\title{
Verification of the CFD calculation for the centrifugal compressor medium flow model stages with the help of supercomputer
}

\author{
Lyubov Gileva ${ }^{1, *}$, Sergey Kartashov ${ }^{1}$, Anatoliy $Z_{\text {Zuev }}{ }^{1}$, and Vyacheslav Ivanov ${ }^{1}$ \\ ${ }^{1}$ Peter the Great St. Petersburg Polytechnic University, 29 Polytechnicheskaya street, Saint- \\ Petersburg, 195251, Russian Federation
}

\begin{abstract}
The goal of this work is to develop recommendations for the calculating problem formulation of the medium flow centrifugal compressor characteristics by computational fluid dynamics methods with the assessment of the computing resources necessary costs. Calculations are made on supercomputers of SPbPU "Polytechnic" and "DeltaCluster. The object of the research is the centrifugal compressor stage for which the flow investigation has been held in the whole passage. The calculations result comparison with the practical experiment data for the whole working characteristics are shown in this work. The leakage in the lap seals and between the disks gaps investigation work has been made. The calculation of the whole $2 \pi$ flat pattern has been made and also the influence on the calculation results of the between mesh interfaces has been analyzed.
\end{abstract}

\section{Introduction}

Centrifugal compressors (CC) are dynamic action energy machines - tools used to compress and transport gases. The pressure increase is achieved due to the force interaction of the gas flow with rotating and stationary vane equipment. CC are widely used in all basic industries (metallurgy, chemical and petrochemical industry, food industry, etc.), maintenance of pneumatic networks, as well as engaged in the natural gas production, processing, transportation and storage, carry out the reverse injection of associated petroleum gas into the reservoir in oil production. It is obvious that regardless of the machine purpose, the power consumed by a CC is thousands of kilowatts, which increases the requirements for the installation efficiency, and motivates the consumer and the manufacturer to find ways to improve it.

The main efficiency indicator is mainly determined by the energy spent on overcoming the gas dynamic resistance of the flow part. During the recent years, the development of computer, cluster technologies in particular, allowed to come to the computational fluid dynamics methods in the calculation of viscous flows in CC [1,4]. The introduction of numerical simulation in the machine development process can reduce the cost of subsequent experimental refinement, and ideally abandon such at all. Methods of multiparametric

* Corresponding author: 1yubov5reshet@gmail.com 
optimization are also developed, with their help dozens of CC energy-efficient flow parts variants are automatically calculated on the computational cluster [6]. It is also possible to use the complex forms of inlet and outlet chambers for the study, since the flow in them significantly affects the entire flow as a whole [7]. To save the calculation time, also the timeaveraged formulation of the problem is used, so it is impossible to solve non-stationary processes arising in the passage. In addition, there are no recommendations on the accuracy of the computational grids sufficient to calculate a number of the same type stages; turbulence modeling problems are not fully resolved, as well as recommendations on the internetwork interface parameters choice [8-20].

\subsection{Purpose of the work:}

The purpose of the work is to develop recommendations for the stationary calculation problem formulation of the intermediate-type centrifugal compressor stages characteristics (Fig.1) with the help of computational gas dynamics methods with an assessment of the necessary computational resources.

The main research method is numerical simulation based on balance equations iterative solution in Ansys CFX 14.5 Solver. and Ansys CFX 16.2 Solver.



Fig. 1. General view of the research object - intermediate type centrifugal compressor stage

\section{Methods}

The object of the study is the corresponding devices blade sector of two medium-flow centrifugal compressor stages, for which there are experimental characteristics [2]. Flow part of the stage (Fig.2) consists of: axisymmetric inlet chamber (IC), radial impeller of closed type (IM), vane-less diffuser (VLD), back-guide apparatus with straightening blade confuser (BGA) and labyrinth seals (LS) with inter-disc gaps of the hub and shroud disks. Different types of turbulence models were used: Stationary RANS - approach (averaging by Reynolds number) to the solution of Navier-Stokes equations, turbulence model SST (Shear stress transport) [3].

At the diagram (Fig.2) control section 2-2 corresponds to the output of the impeller at a distance of 1.05D2, section 3-3 corresponds to the output of vane-less diffuser, section 0'-0' corresponds to the output of the stage. For the impeller stage №1 the rotating area with the velocity of $n=9840 \mathrm{rpm}$ was set, for stage №2 $\mathrm{n}=10020 \mathrm{rpm}$. 




Fig. 2. Control sections, stage intermediate elements and boundary conditions scheme
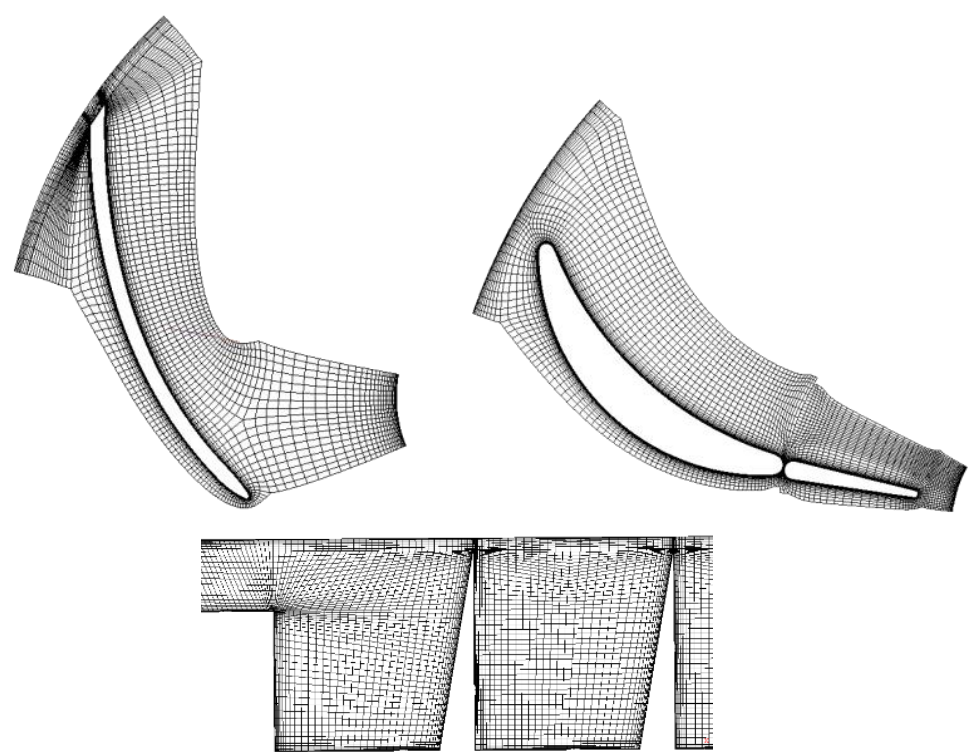

a)

b)

C)

Fig. 3. Stage elements grid view for: a) impeller, b) back-guide apparatus, C) axial labyrinth seal

\section{Results and Discussion}

\subsection{Calculation results verification}

The calculation for verification was carried out with the "stage" interface in the entire calculation area. The graphs on Fig. 4, 5 show a good agreement with the calculation results of the experiment in the zone of optimal mode $\mathrm{f}=0.062$ for stage №1, $\mathrm{f}=0.055$ for stage №2 and is in the field of engineering error, but when moving to the zone of maximum mas flow the error increases significantly. Errors arise due to the low-energy flow separation zones associated with the bad flow conditions of blades deterioration in these modes, therefore, there is performance overestimation, as the calculation results shows that the flow separation is missing, so there is no energy loss. On the graphs of Fig. 6, 7 it can be seen that the loss coefficient for the stage elements qualitatively coincides, except for vane-less diffuser in the zone of maximum mass flow. A large error in this zone is due to the reasons described above. To solve this problem, the solution of different turbulence models and corresponding 
computational grids should be evaluated. The plank is installed on the experimental dependencies, which represents the relative acceptable engineering accuracy of the experiment $\pm 2,5 \%$.

Polytropic efficiency coefficient by total parameters and the pressure ratio in the control sections were determined by the equations:

$$
\eta_{p}^{*}=h_{p}^{*} / h_{i}, \quad \Pi=P_{i-i} / P_{0-0} .
$$


Fig.4. The calculated and experimental stage characteristics № 1. Efficiency by total parameters and the pressure ratio for three control cross sections 

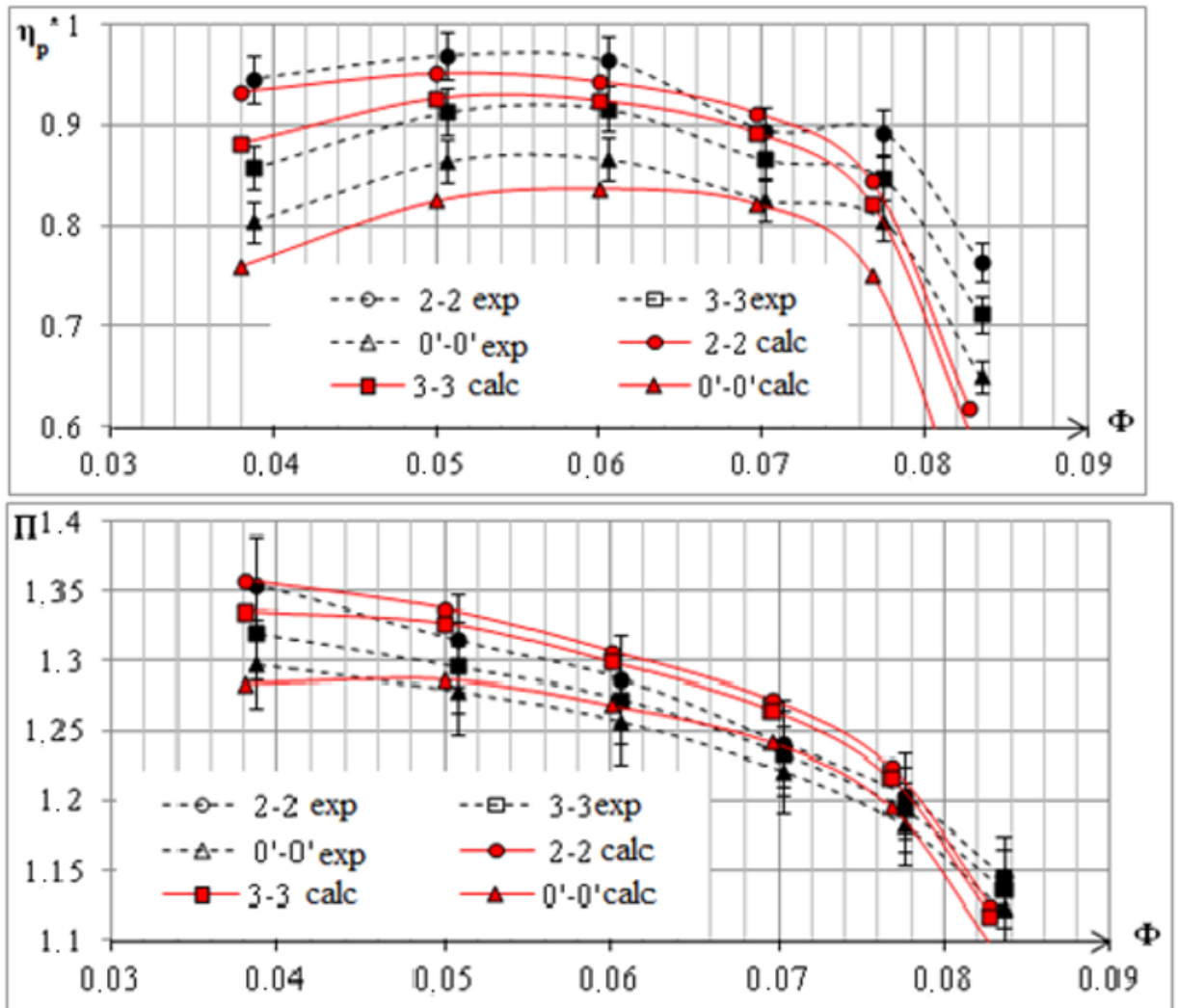

Fig.5. The calculated and experimental stage characteristics № 2. Efficiency by total parameters and the pressure ratio for three control cross sections


Fig.6. Characteristics the coefficient of the total pressure at the test section level 1 (upper) and stage 2 (bottom) 


\section{EECE-2018}
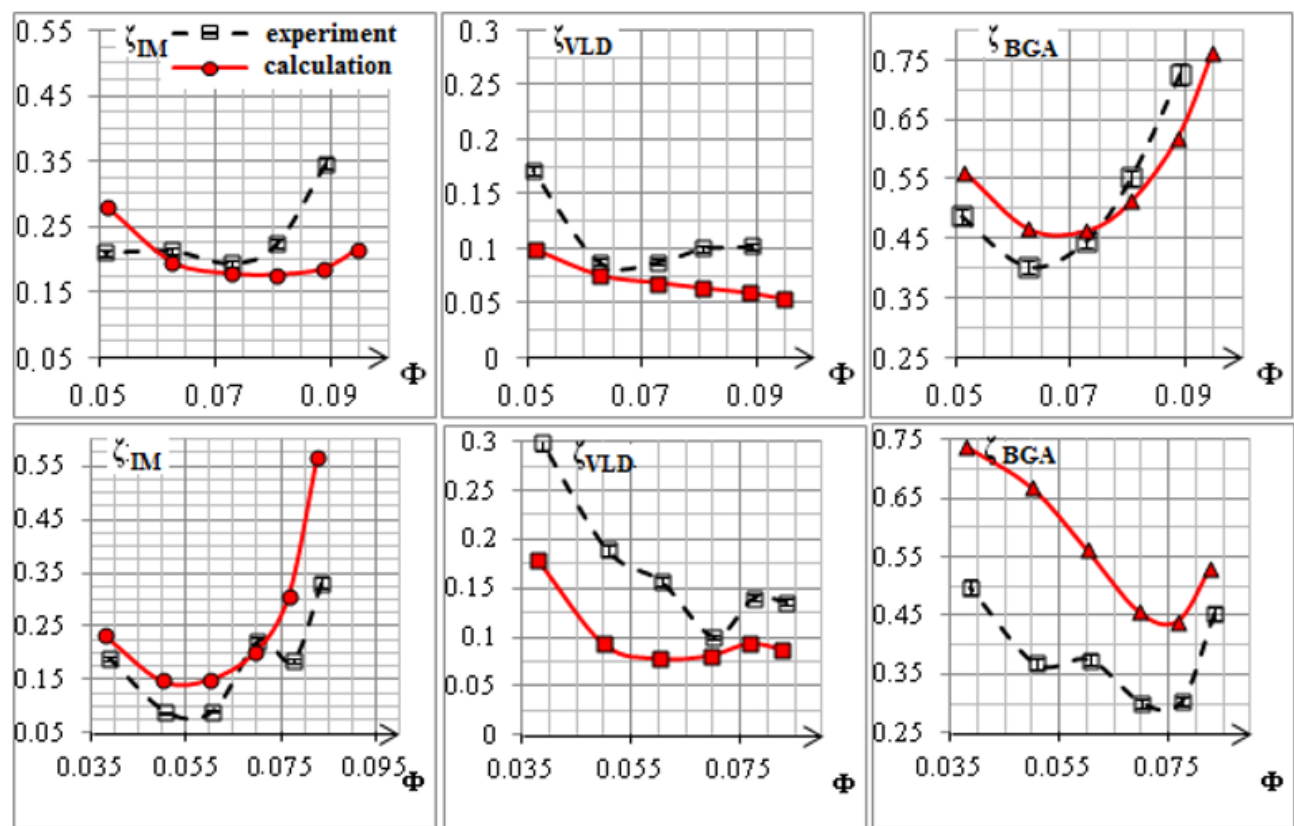

Fig.7. Loss coefficient characteristics graphs in the stage 1 elements (top) and stage 2 (bottom)

\subsection{Interfaces influence analyses}

For fig. 8 it is shown that the application of the interface "stage" in the entire computational domain (between the inlet chamber and the impeller, the impeller and vane-less diffuser, vane-less diffuser and back-guide apparatus) led to a minimum error with the experiment, the greatest error is obtained by using the interface "frozen rotor". Also, the average version of "Mix" with the interface"stage" was calculated only at the inlet and outlet of the impeller, the calculation of the characteristics showed that in the section $0^{\prime}-0^{\prime}$ the characteristic is overestimated and decreases from right to left in the range from $4 \%$ to $1.7 \%$, in the other sections the characteristic are identical.



Fig.8. Polytropic efficiency characteristics Graph by total parameters at the control sections for different inter-grid interfaces 


\subsection{Gaps and seals influence analysis}

Disconnection from the calculated area of inter-disc gaps and labyrinth seals has led to the expected increase in the efficiency by $2.2 \%$ in the cross section $2-2$ (Fig.9), and by $1.6 \%$ $1.7 \%$ in the output section $0^{\prime}-0$ ', except for one point, which is due to poor convergence in low-performance modes, as non-stationary processes are taking place. The calculation was carried out with the "stage" interface in the entire calculation area.



Fig.9. Polytrophic efficiency characteristics graph by total parameters in the control sections when seals are disconnected from the computational domain for stage 1

The mass fraction of leaks is determined by the equation:

$$
\begin{gathered}
\beta_{\text {leak }}=\frac{\bar{m}_{\text {leak }}}{\bar{m}} \cdot 100 \% \\
\beta_{\text {leak }}^{\text {shroud }}=\frac{0.0168}{1.938} \cdot 100 \%=0.87 \%, \beta_{\text {leak }}^{\text {hub }}=\frac{0.0056}{1.938} \cdot 100 \%=0.29 \%
\end{gathered}
$$

\section{Conclusions}

Centrifugal compressor stages characteristics modeling qualitatively and quantitatively coincides with the field of real experiment in the bounds of acceptable error in engineering calculations up to $\pm 2.5 \%$ in the zone of optimum performance. For other modes, additional other turbulence models and methods of numerical solution formulation should be investigated, solving low-energy loss associated with flow separation, as well as emerging unsteady processes.

This research was supported by Compressor vacuum and refrigeration engineering department administration. We thank our colleagues from Compressor vacuum and refrigeration engineering department, who provided insight and expertise that greatly assisted the research.

\section{References}

1. Y.B. Galerkin, Y.V. Kozhukhov, A.M. Danilishin, Week of Science SPBSTU, 17-20 (2013).

2. Y.B. Galerkin, Proceedings of the scientific school of compressor SPbSPU, 188-210 (2010)

3. G.A. Gerolymos, Vallet, AIAA Journal, vol. 40, (2002). 
4. A.M. Yablokov, Yu.V. Kozhukhov, A.A. Lebedev, St. Petersburg State Polytechnic University Journal of Engineering Science and Technology, 4 (231), 59 - 69 (2015).

5. Y. Boldyrev, A. Rubtsov, Y. Kozhukhov, A. Lebedev, I. Cheglakov, A. Danilishin, CEUR Workshop Proceedings. Volume 1482, 273-279. (2015)

6. Y.V. Kozhukhov, A.M. Danilishin, V.K., IOP Conference Series Materials Science and Engineering 08/2015; 90(1):012046. (2015). DOI:10.1088/1757-899X/90/1/012046

7. Y.V. Kozhukhov, V.K. Yun, L.V. Reshetnikova, M.V. Prokopovich, IOP Conference Series Materials Science and Engineering, 90(1):012047 August 2015. DOI: 10.1088/1757-899X/90/1/012047 (2015).

8. C.M. Anderson, Ö.F. Turan, B. Brzek, L. Castillo, 15th Australasian Fluid Mechanics Conference The University of Sydney, Sydney, Australia December (2004).

9. F. Bet, G. Seider Dynamics magazine, 23-26 (2014)

10. G. Sravan Kumar, Dr. D. Azad and K. Mohan Laxmi. International Journal of Mechanical Engineering and Technology, 7(6), 634-641 (2016)

11. Z. Liu, and D. L. Hill, International Compressor Engineering Conference, Paper 1369 (2000).

12. M. P. King, M. Wilson, and J. M. Owen, Proc. ASME Turbo Expo, paper GT2005-68948. (2005)

13. B. Rosic, J.D. Denton, and G. Pullan, Proc. ASME Turbo Expo, paper GT2005-68459. (2005)

14. J.W. Chew, S. Dadkhah, and A.B. Turner, ASME J. Turbomachinery, 124, 306-315 (1992)

15. M.P. Wernet, M.M. Bright, and G.J. Skoch. An investigation of surge in a high-speed centrifugal compressor using digital PIV. Paper TM2002-211832, NASA, (2002).

16. D.E. van Zante, A.J. Strazisar, J.R. Wood, M.D. Hathaway, and T.D. Okiishi. Recommendations for achieving accurate numerical simulation of tip clearance flows in transonic compressor rotors. Paper TM2000-210347, NASA, (2000).

17. W. Zhao, J. Sheng, J. Yang, J. Song, Transactions of the Chinese Society of Agricultural Engineering (Transactions of the CSAE). 6 (2015)

18. F. Zhang, R. Baar. ICMAA, 8 108, 08012, (2017) DOI: 10.1051/ 71080

19. S. Rane, A. Kovačević, N. Stošić, M. Kethidi, 8th Int conf on compressors and their systems, C1390, (2013)

20. D.A. Roberts and R. Steed. 2004 ANSYS CFX conference (2004). 